\title{
DIAGNOSTIC ACCURACY OF COMPUTED TOMOGRAPHY IN THE PRE-OPERATIVE EVALUATION OF POSTERIOR FOSSA TUMOURS IN CHILDREN
}

\author{
SARKER T ${ }^{1}$, SARKER RN $^{2}$, RABBI AF $^{3}$, DAS K $^{4}$, HOSSAIN A $^{5}$
}

\begin{abstract}
Objective: To evaluate the diagnostic accuracy of computed tomography $(C T)$ in the pre-operative evaluation of posterior fossa tumours in children.

Materials and Methods: CT scan of brain was done on 30 consecutively selected children with clinical suspicion of posterior fossa tumours and they were followed up to the final diagnosis by histopathology. The diagnostic accuracy, sensitivity, specificity, positive and negative predictive values of the procedure was calculated for different lesions.

Results: Out of the 30 patients, 17 (56.67\%) had medulloblastoma, 8 (26.67\%) were diagnosed as astrocytoma, 3 (10\%) as ependymoma and 2 (6.66\%) as brain stem glioma. 28 cases were accurately diagnosed by $C T$ and 2 were misdiagnosed. The sensitivity, specificity, accuracy, positive predictive value and negative predictive value of CT were.88.89\%, 100\%, 96.67\%, $100 \%$ \& 95.54\% respectively for astrocytoma; $100 \%, 92.86 \%, 96.67 \% \%, 94.12 \% \& 100 \%$ respectively for medulloblastoma; 66.67\%, 96.3\%, 93.33\%, 96.3\% \&66.67\% respectively for ependymoma and $100 \%$ each for brainstem glioma.

Conclusion: $C T$ is a highly sensitive and specific procedure for the diagnosis of posterior fossa tumours in children. So it can be regarded as a primary imaging modality of different pediatric posterior fossa tumours.
\end{abstract}

Keywords: Diagnostic accuracy, computed tomography, posterior fossa tumours.

J Dhaka Med Coll. 2012; 21(2) : 188-196.

\section{Introduction}

Brain tumours are the most common neoplasm in children next to leukemia with an incidence of approximately 2.5 per 100,000 per years. ${ }^{1}$ Seventy percent of childhood CNS tumors arise in posterior cranial fossa. ${ }^{2}$ The most common lesions are intra-axial tumors such as cerebellar astrocytomas, medulloblastomas, brain stem gliomas and ependymomas Extra axial tumour such as chordomas and schwannomas are rare but not uncommon.

From the mid and the late 1970s, CT emerged as the primary diagnostic screening modality for the detection of intracranial pathology. ${ }^{3} \mathrm{CT}$ is highly accurate in delineation and characterization of new and recurrent tumours. ${ }^{4-5}$ It assess the site of primary lesion, size, extent of involvement, precontrast density (cystic or solid), post-contrast enhancement, regularity of borders, and presence of calcification. CT also demonstrates the presence of edema, deformity and displacement of the fourth ventricle, cisternal deformity, bone erosion and widening of the cerebral ventricles as reflected by an increased cerebroventricular index. ${ }^{6-7}$ Vascular structures can

1. Dr. Taposhi Sarker, Senior Registrar, Department of Radiology \& Imaging, Bangladesh Institute of Health Sciences (BIHS) and Hospital, Dhaka.

2. Dr. Robindra Nath Sarker, Assistant Professor, Department of Radiology \& Imaging, Dhaka Medical College and Hospital, Dhaka.

3. Akanda Fazle Rabbi, Medical Officer, Department of Radiology \& Imaging, Dhaka Medical College Hospital, Dhaka.

4. Dr. Kanta Das, Consultant, Department of Radiology \& Imaging, BIRDEM Hospital, Dhaka.

5. Prof. Ahmed Hossain, Professor and Head, Department of Radiology \& Imaging, Dhaka Community Medical College and Hospital, Dhaka.

Correspondence: Dr. Taposhi Sarker, Department of Radiology and Imaging, Bangladesh Institute of Health Sciences (BIHS) and Hospital, Dhaka. Cell Phone: +8801718356132, E-mail: dr.taposhi@ yahoo.com 
be further defined with the help of contrast and vascular structures other than vessels can be determined.

CT has made a significant impact on the differential diagnosis of intracranial tumours, particularly those which developed in the posterior cranial fossa in infants and children. Subarachnoid spread (supratentorial, infratentorial \& spinal subarachnoid space) can be seen in medulloblastoma on the initial CT and on post operative follow-up. Again early CT diagnosis depends on displacement of the fourth ventricle and compression and distortion of cisterns. Mass effect and cisternal compression or obliteration can be appreciated in nearly every case of brain stem glioma. Although bone artifacts have been an obstacle to the optimal demonstration of posterior fossa contents, the diagnosis obtained with $\mathrm{CT}$ is reasonably accurate. ${ }^{8-9}$ MRI has its advantage of being radiation free, multiplanner imaging facility hence a lesion can be seen in three planes and accurate localization of the lesion can be done, better soft tissue characterization and can clearly demonstrate peritumoral edema, but its disadvantage includes its high cost and paucity of its availability. MRI is only available in limited number of centers in many developing countries like Bangladesh. For this reason MRI is not yet widely used. It also requires long time for acquisition of the data and very difficult to use for restless patients particularly in neonates and infants and even in uncooperative children and cannot be done in patients having metallic implants for which CT is the modality of choice. Hence, CT has become the most commonly used primary radiologic investigation for posterior cranial fossa tumors. Iodinated contrast media is used to visualize all types of posterior cranial fossa tumours.

In posterior fossa tumors, coronal images are often extremely helpful for evaluating the relationship of a tumor to the tentorium and the foramen magnum. Coronal images are also helpful in the evaluation of small lesions situated near dura or bone. Reformatted sagittal images may aid in evaluation of tumors of the midline structures such as the third ventricle.
Newer multi-slice helical CT scanners are capable of providing highly collimated sub millimeter thickness sectional images in extremely short acquisition times and thus areas of hyperostosis or bone destruction, intratumoral calcification and early intratumoral or peritumoral hemorrhage are more completely defined with greater certainty on CT than on MRI . ${ }^{10}$

$\mathrm{CT}$ is often more specific than MR imaging for preoperative tumour diagnosis. For example, small round cell tumours such as germinomas and medulloblastomas are isodense or hyperdense compared with brain parenchyma prior to administration of contrast agent, whereas astrocytomas of childhood are almost always hypo dense. Thus, in general, germinomas can be confidently differentiated from astrocytomas with use of CT. Making this differentiation on the imaging study alone is often not possible with MR imaging. Similarly, the presence of calcification, which can be helpful in the preoperative diagnosis of ependymoma and teratomas, is less easily detected by MR imaging than by CT.

There have been many works on the role of CT in the diagnosis of posterior cranial fossa tumor like medulloblastoma, cerebellar astrocytoma, ependymoma, brain stem glioma, cerebellopontine angle (CPA) tumour, but however few works have been done including all types of posterior cranial fossa tumors specially in children. Such types of study have not been done yet in our country. Hence, certainly this work will serve as a baseline study for other researchers. After having multicenter study a definite level will be achieved for a conclusive recommendation regarding $\mathrm{CT}$ evaluation of pediatric posterior fossa tumours. The present study was thus designed to assess the efficacy of CT for evaluation of different pediatric posterior fossa tumours.

\section{Materials and Methods}

This prospective study was carried out from July 2006 to February 2007 in the Department of Radiology \& Imaging, Dhaka Medical College Hospital (DMCH) and Bangabandhu Sheikh Mujib Medical University (BSMMU) Hospital on consecutively selected 35 patients ranging 
from 1 to 15 years of age who were admitted into the Department of Neurosurgery of the respective hospitals with clinical suspicion of posterior fossa tumours and were referred for CT scan of brain. CT scan of brain was performed in all cases. Among 35 cases, 2 cases were diagnosed as arachnoid cyst, 1 case as Dandy-Walker variant; 1 patient had clinical signs of infection and a low density lesion in the cerebellum compatible with cerebellitis and another patient with leukemia had a hyperdense homogeneously enhanced lesion at the petrous apex. Histopathological examination was not done in these 5 cases. Hence, these 5 cases were excluded from this study. The rest 30 patients underwent surgery and were followed up to histopathological diagnosis. Then CT diagnoses were compared with histopathological diagnoses. Statistical analysis was done to see the diagnostic accuracy of CT for the diagnosis of posterior fossa tumors by calculating sensitivity, specificity, accuracy, positive and negative predictive values for different lesions separately.

Prior to commencement of this study, the research protocol was approved by the appropriate authority and written informed consent was obtained from the patients or their parents. CT scan of brain was performed from caudal to cephalad level with 15 to 20 degree angulation to the canthomeatal line both before and after the I.V contrast agent (Iopamidol-370) with $5 \mathrm{~mm}$ to $10 \mathrm{~mm}$ slice thickness at $120 \mathrm{Kv}$ and $150 \mathrm{~mA}$ and were viewed in axial and if needed in coronal slices. CT scans were done after proper counseling of the patient and after proper sedation of restless children.

\section{Observations and Results}

The age of the patients ranged between 1 to 15 years with the mean age of 9.07 years. Highest incidence was found in the age group of 10 to 12 years. 20 were male $(66.67 \%)$ and 10 were female $(33.33 \%)$. The most common symptom was headache which was observed in 27 patients (90\%). 21 patients (70\%) had vomiting and 10 patients (33.3\%) had gait disturbance. Blurring of vision was noted in 8 patients (26.7\%). Convulsion was present in 4 patients (13.3\%). Weakness of limb was observed in 3 patients $(10 \%)$. Vertigo was found in 12 patients (40\%). Enlarged head was present in 1 patient $(3.33 \%)$ and 2 patients $(6.67 \%)$ had altered consciousness.

Table-I

CT characteristics of the posterior fossa tumours

\begin{tabular}{|c|c|c|c|c|c|c|c|c|c|}
\hline \multirow[t]{2}{*}{ Characteristics } & & \multicolumn{2}{|c|}{$\begin{array}{l}\text { Medulloblastoma } \\
\qquad(\mathrm{n}=17)\end{array}$} & \multicolumn{2}{|c|}{$\begin{array}{l}\text { Astrocytoma } \\
(\mathrm{n}=8)\end{array}$} & \multicolumn{2}{|c|}{$\begin{array}{l}\text { Ependymoma } \\
\qquad(\mathrm{n}=3)\end{array}$} & \multicolumn{2}{|c|}{$\begin{array}{c}\text { Brain stem } \\
\text { glaioma } \\
(\mathrm{n}=2)\end{array}$} \\
\hline & & No. & $\%$ & No. & $\%$ & No. & $\%$ & No. & $\%$ \\
\hline \multirow[t]{2}{*}{ Borders } & Regular & & - & 1 & 12 & - & & - & \\
\hline & Irregular & 17 & 100 & 7 & 87 & 3 & 100 & 2 & 100 \\
\hline \multicolumn{2}{|l|}{ Calcification } & 2 & 11 & 1 & 12 & 2 & 66 & - & \\
\hline \multicolumn{2}{|c|}{ Peritumoral oedema } & 15 & 88 & 5 & 62 & 3 & 100 & 1 & 50 \\
\hline \multicolumn{2}{|c|}{ Compressed fourth ventricle } & 17 & 100 & 7 & 87 & 3 & 100 & 2 & 100 \\
\hline \multicolumn{2}{|c|}{ Location within fourth ventricle } & & - & - & & 2 & 66 & - & \\
\hline \multicolumn{2}{|l|}{ Hydrocephalus } & 15 & 88 & 6 & 75 & 2 & 66 & 1 & 50 \\
\hline \multirow[t]{4}{*}{ Density } & Mixed & 2 & 11 & 2 & 25 & 1 & 33 & - & \\
\hline & Hypodensity & & - & 5 & 62 & - & & 1 & 50 \\
\hline & Hyperdensity & 10 & 58 & - & & - & & - & \\
\hline & Isodensity & 5 & 29 & 1 & 12 & 2 & 66 & 1 & 50 \\
\hline \multirow[t]{2}{*}{ Enhancement } & Heterogeneous & 3 & 17 & 7 & 87 & 3 & 100 & 2 & 100 \\
\hline & Homogeneous & 14 & 82 & 1 & 12 & - & & - & \\
\hline
\end{tabular}


Table- II

CT and histopathological diagnoses of the posterior fossa tmours

\begin{tabular}{lcc}
\hline Diagnoses & $\begin{array}{c}\text { No. and percentage } \\
\text { of patients diagnosed by CT }\end{array}$ & $\begin{array}{c}\text { No. and percentage } \\
\text { of patients diagnosed by } \\
\text { histopathology }\end{array}$ \\
\hline Medulloblastoma & $17(56.67 \%)$ & $16(53.33 \%)$ \\
Astrocytoma & $8(26.67 \%)$ & $9(30 \%)$ \\
Ependymoma & $3(10 \%)$ & $3(10 \%)$ \\
Brainstem glioma & $2(6.66 \%)$ & $2(6.67 \%)$ \\
Total & $30(100 \%)$ & $30(100 \%)$ \\
\hline
\end{tabular}

Table-III

Measures of diagnostic performance of CT

\begin{tabular}{lcccc}
\hline Index & $\begin{array}{c}\text { Cerebellar } \\
\text { astrocytoma. }\end{array}$ & Medulloblastoma. & Ependymoma & $\begin{array}{c}\text { Brainstem } \\
\text { glioma }\end{array}$ \\
\hline Sensitivity & $88.89 \%$ & $100 \%$ & $66.67 \%$ & $100 \%$ \\
Specificity & $100 \%$ & $92.86 \%$ & $96.3 \%$ & $100 \%$ \\
Accuracy & $96.67 \%$ & $96.67 \%$ & $93.33 \%$ & $100 \%$ \\
Positive predictive value & $100 \%$ & $94.12 \%$ & $96.3 \%$ & $100 \%$ \\
Negative predictive value & $95.45 \%$ & $100 \%$ & $66.67 \%$ & $100 \%$ \\
\hline
\end{tabular}

Out of the 30 cases, CT diagnosed 28 cases correctly and 2 cases were misdiagnosed. CT misdiagnosed one case of astrocytoma as ependymoma and one case of ependymoma as

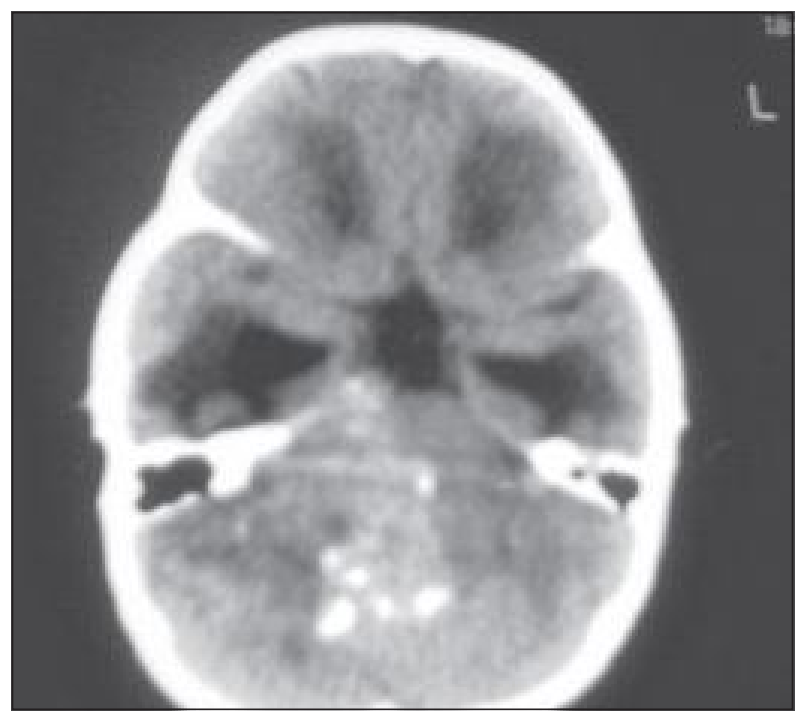

Fig.-1: NECT of brain showing mixed density cerebellar astrocytoma having nodular calcification. medulloblastoma. Measures of diagnostic performance of $\mathrm{CT}$ in different posterior fossa tumors are shown in table III.

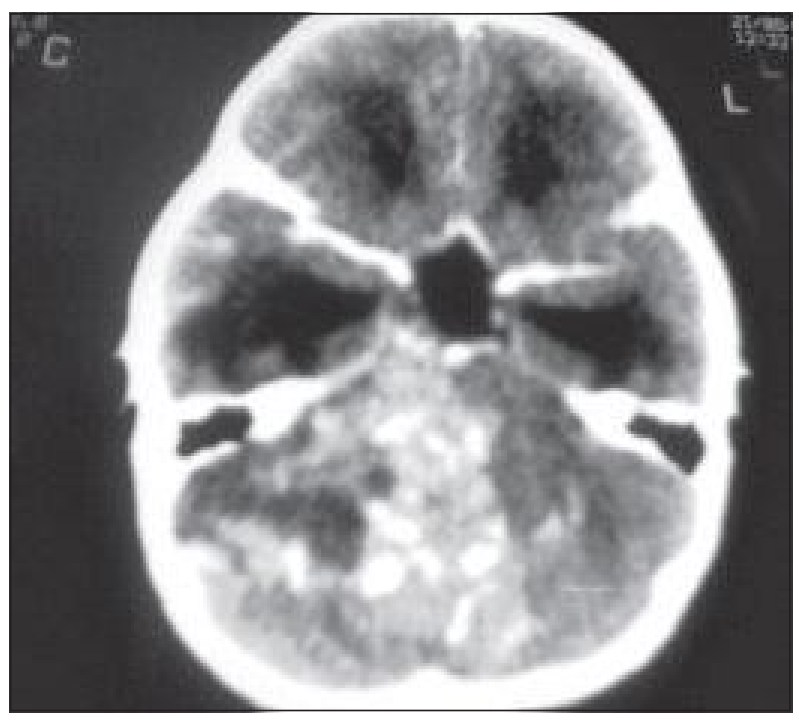

Fig.-2: CECT of brain showing heterogeneously enhancing Cerebellar astrocytoma. 


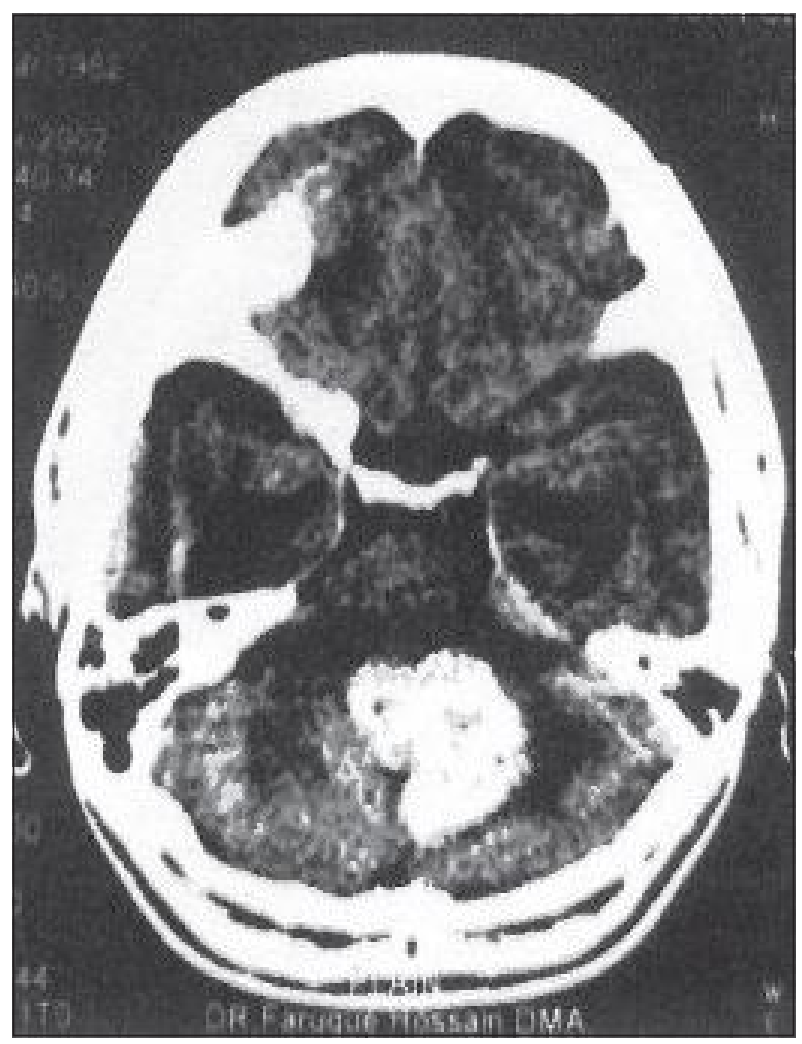

Fig.-3: NECT of brain showing hyperdense medulloblastoma.

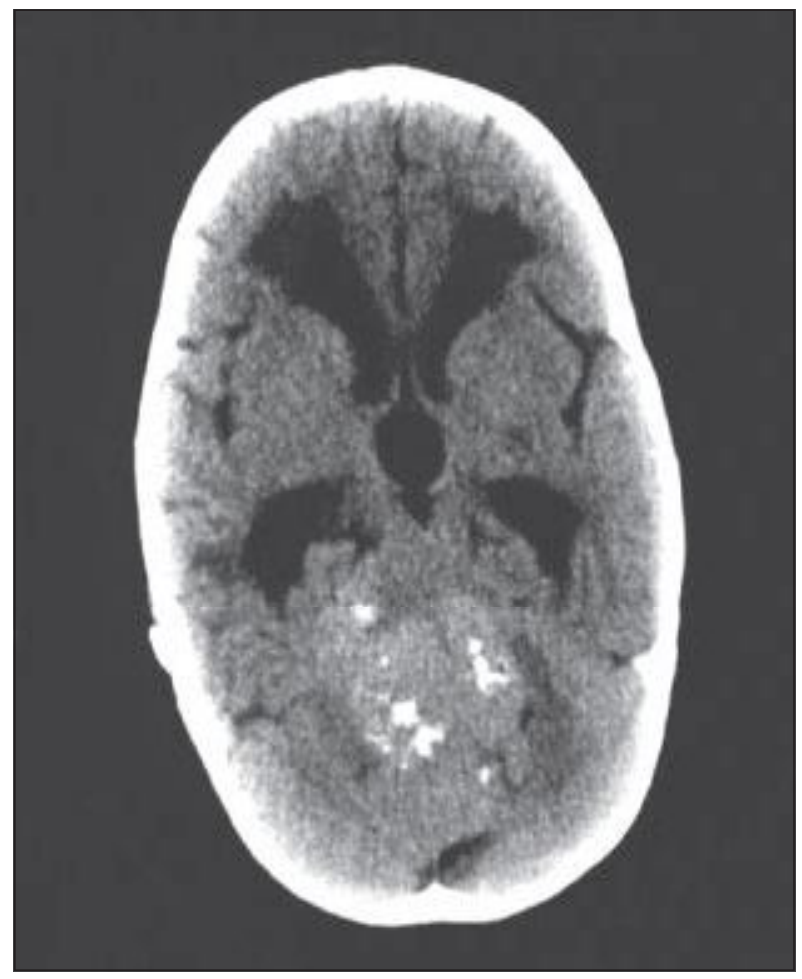

Fig.-5: NECT Scan of brain showing isodense ependymoma with calcification.

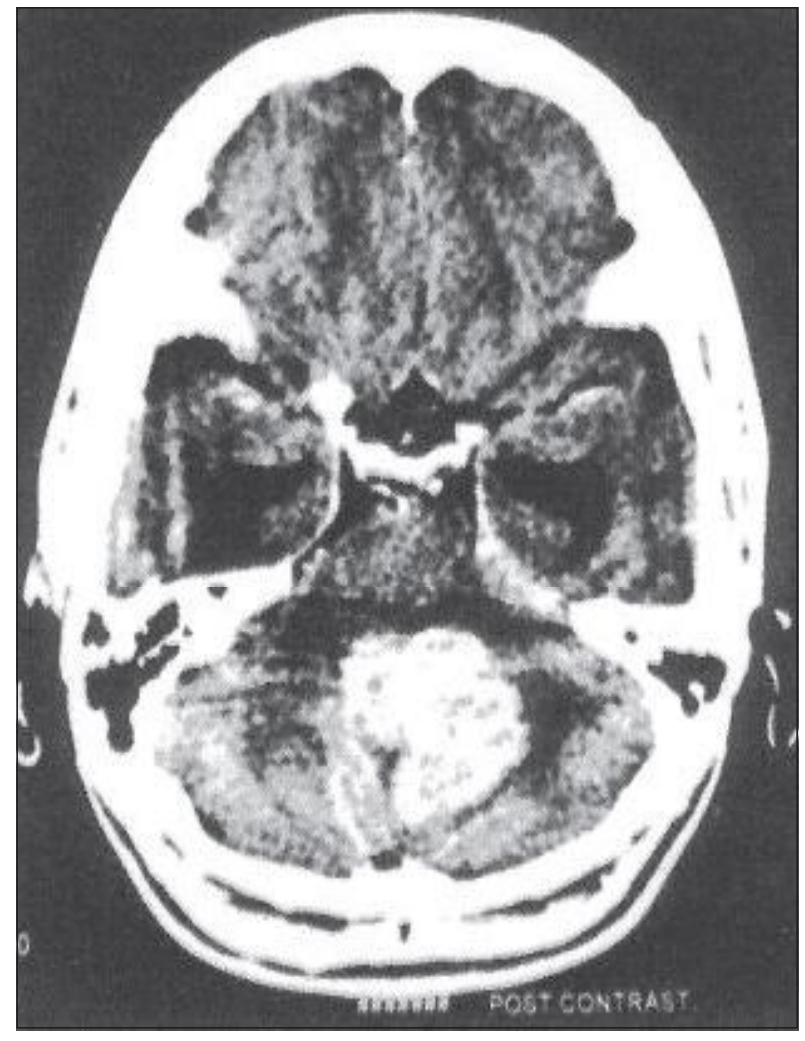

Fig.-4: CECT of brain showing enhancing medulloblastoma.

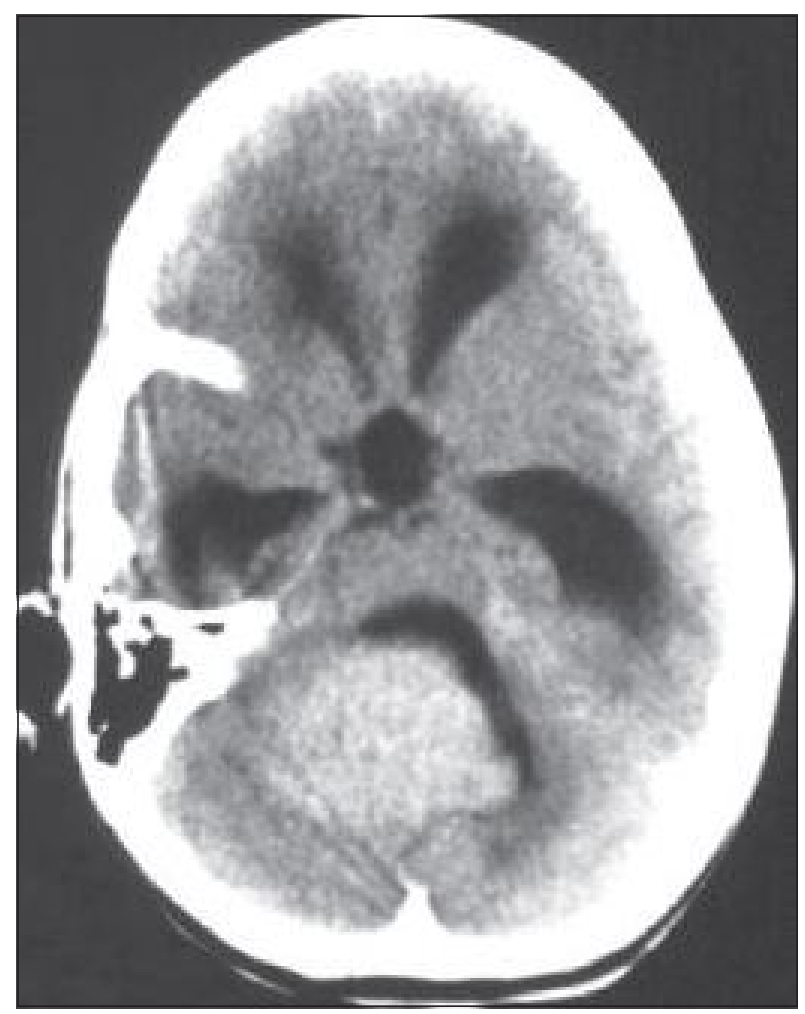

Fig.-6: CECT Scan of brain showing enhancing ependymoma. 


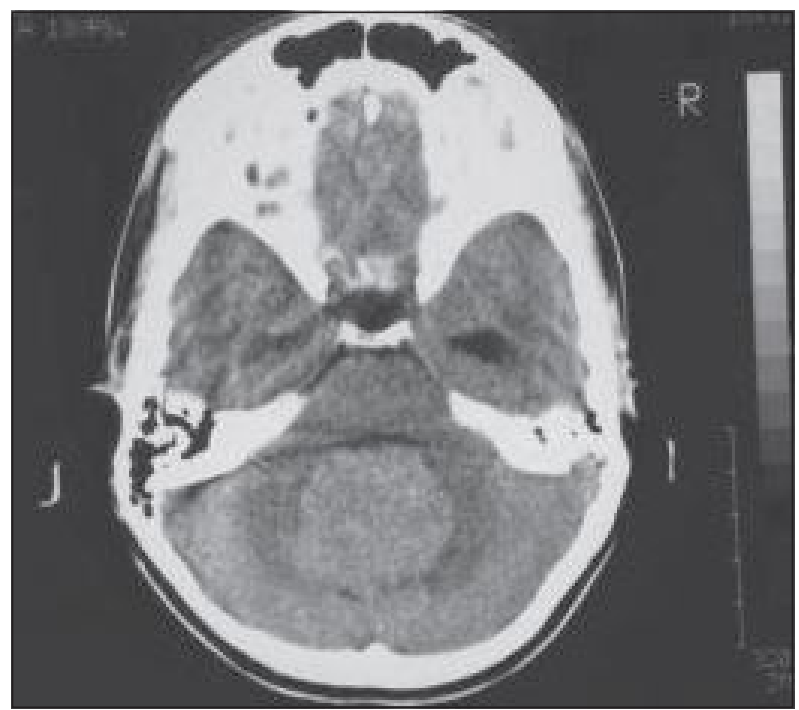

Fig.-7: NECT of brain showing hyperdense medulloblastoma.

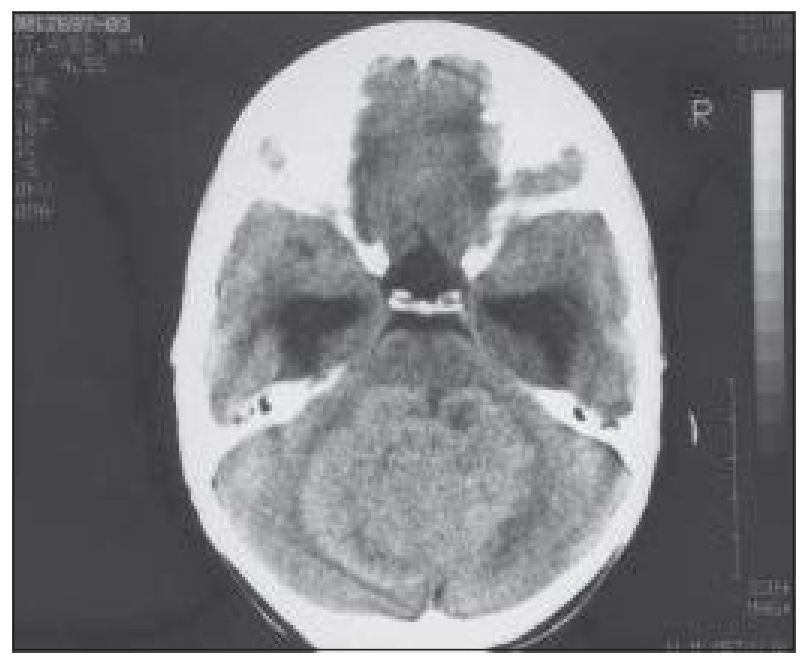

Fig.-9: NECT Scan of brain showing mixed density ependymoma.

\section{Discussion}

Computed tomography has revolutionized the cross sectional imaging, it provides detailed anatomical outline without adjacent structures interfering with the visualization of each other. It is the primary imaging modality in the evaluation of brain tumour. In most cases, the exact diagnosis should be made by histopathological examination but it is important for treatment planning that the appropriate depiction of tumour extension and tissue characterization can be made by CT.

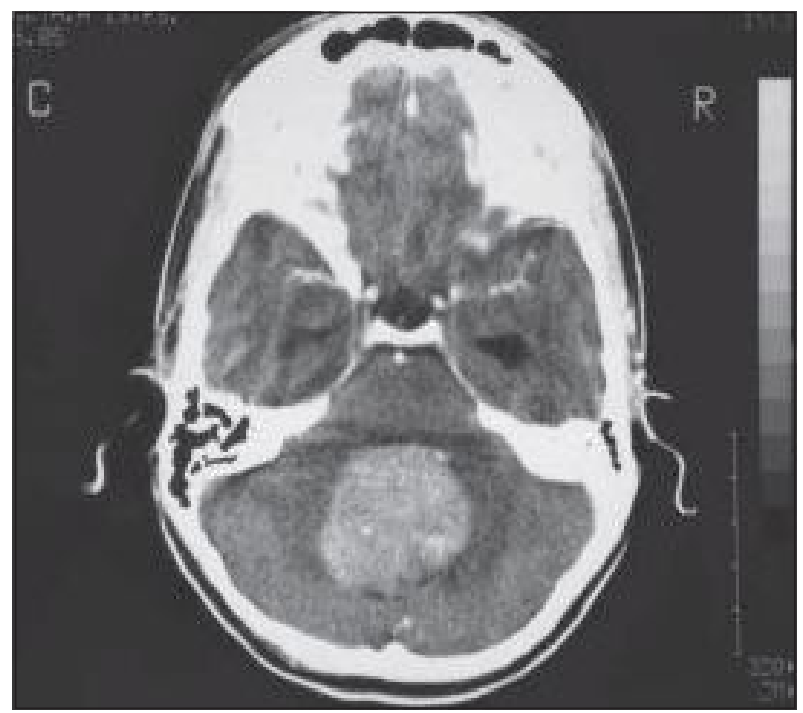

Fig.-8: CECT of brain showing enhancing medulloblastoma.

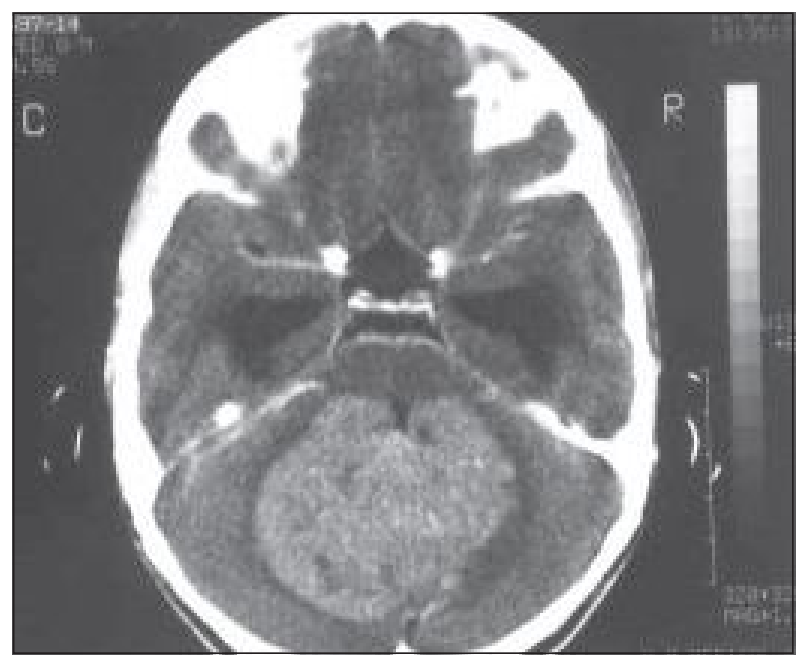

Fig.-10: CECT Scan of brain showing enhancing ependymoma.

On CT images, the typical juvenile pilocytic astrocytoma (JPA) appears as a smoothly marginated, hypodense cyst like mass with a well defined, less hypodense tumour nodule on one wall. The nodule may contain one or more areas of dense calcification. The cyst fluid is less hypodense than the CSF (15-25 HU) because of the high protein concentration. Typically, after intravenous administration of a contrast agent, dense homogeneous contrast enhancement of the tumor nodule, but not of the other walls of the cyst, is seen. On CT scan, 
low grade diffuse cerebellar astrocytoma appears most commonly as poorly marginated areas of decreased attenuation. Contrast enhancement is usually absent. On CT scan, low grade diffuse cerebellar astrocytoma appears most commonly as poorly marginated areas of decreased attenuation. Contrast enhancement is usually absent.

On non-contrast CT images, medulloblastomas appear as rounded or oblong, mainly homogeneous, isodense to slightly hyperdense masses centrally located in the inferior vermis and cavity of the fourth ventricle. Obstruction of the fourth ventricle, inferior aqueduct or both with resulting enlargement of the third and lateral ventricles is commonly seen on CT at the time of clinical presentation. Punctate or nodular intratumoral calcifications are identified in $10 \%$ to $20 \%$ of cases. Hypodense intratumoral foci of necrosis and cysts are found in up to $50 \%$ of medulloblastoma. After intravenous injection of contrast medium, moderate to intense homogenous enhancement of the solid tumor mass is the rule in these tumours, although approximately $10 \%$ fail to demonstrate contrast enhancement on CT. ${ }^{3}$

The CT diagnosis of brainstem tumor is based on two factors: a) the appearance of the CSF structures adjacent to the brain stem, and b) the parenchymal density before and after contrast enhancement. Appreciation of the distortion or obliteration of the fourth ventricle and/or the adjacent cisterns is of utmost importance. A large proportion of the tumours are isodense relative to the normal brain parenchyma and present as enlargement of the brainstem, on occasion occurring with hydrocephalus. ${ }^{11}$

Diffuse enlargement of all or part of the brainstem is the most common finding in CT. These tumours appear on non-contrast CT as poorly marginated isodense or slightly hypodense masses. Approximately 50\% of brainstem gliomas exhibit some degree of contrast enhancement on CT and MR images following intravenous injection of contrast media. Enhancement is usually patchy and heterogeneous and does not appear to correlate with tumour histology.
In non-contrast $\mathrm{CT}$, ependymomas appear as hypodense or isodense, heterogeneous, midline, rounded masses within the fourth ventricle that partially or completely obliterate it. The tumour is typically well defined by a prominent hypodense halo of peritumoral oedema. Aggregates of calcification, usually small and round but sometimes quite large, are found in up to half of these lesions. Following intravenous contrast injection, the solid portions and cyst walls of the tumour mass exhibit moderate but heterogeneous enhancement. Extension into the lateral recesses, cerebellopontine cisterns or vallecula is most accurately delineated on post contrast $\mathrm{CT} .^{3}$

Various reports on posterior cranial fossa tumours in children confirm that medulloblastoma, cerebellar astrocytoma, brain stem glioma and ependymoma are the common lesions. In this present study, common tumors were medulloblastoma (56.67\%), astrocytoma (26.67\%), ependymoma (10\%) and brain stem glioma (6.66\%). These findings were close to the study of Chang et al. ${ }^{12}$ who found medulloblastoma $40 \%$, followed by astrocytoma $23 \%$, brain stem glioma $21 \%$ and ependymoma $11 \%$. However, in our study incidence of ependymoma is greater than brain stem glioma. Medulloblastomas were more common in the series of Kingsley and Kendall ${ }^{8}$ and Segall et al. ${ }^{13}$ which are similar to the present study. Astrocytomas were the most frequent tumors in the series of ReiderGroswasser et al. ${ }^{7}$, as well as in the series of Naidich et al. ${ }^{14}$. However, our figure showed that astrocytomas were second to medulloblastomas. According to the reports of Lee et al. ${ }^{15}$ and Kingsley and Kendall ${ }^{8}$, medulloblastoma is more frequent in boys, who account for $61.5-88 \%$, cerebellar astrocytoma and brain stem glioma involve both sexes equally, but ependymoma shows a female preponderance of $80 \%$. This study results were somewhat different: boys predominated in the medulloblastomas (56\%), brain stem glioma $(100 \%)$ and ependymomas (66\%), while cerebellar astrocytoma involved both sexes equally with a slight $(55 \%)$ female predominance. 
In this study, in non-contrast CT (NECT) astrocytomas were hypondense in $62 \%$, isodense in $12 \%$ cases, and mixed density in $25 \%$ cases. Calcification was present in $12 \%$ cases. Change et al. ${ }^{12}$ showed that astrocytoma was hypondense in $57 \%$, isodense in 23\%, mixed density in $13 \%$ and hyperdense in $7 \%$ cases. Calcification was present in $17 \%$ cases. In this study, cases showed contrast enhancement which was supported by Chang et al. ${ }^{12}$ In NECT $58 \%$ of medulloblastoma were hyperdense, $29 \%$ were isodense. Hydrocephalus was seen in $69 \%$ cases. Homogeneous contrast enhancement was present in $82 \%$ cases. These findings were similar to Weisburg ${ }^{16}$ who have mentioned 30\% of medulloblastoma were isodense, $70 \%$ hyperdense and $100 \%$ cases hydrocephalus in precontrast scan, showed homogenous contrast enhancement in $90 \%$ cases.

Most of the ependymomas were in the fourth ventricle, therefore being midline ${ }^{12}$ and another reports of Segall et al. ${ }^{13}$ showed about three fifths of ependymomas have extended through the lateral recess of the fourth ventricle and the adjacent tissue into the cerebellopontine angle cistern on one or both sides; in our study one third tumors were laterally located. According to the reports of Kingsley and Kendall ${ }^{8}$ and Segall et al. ${ }^{13}$, calcification was found most often in ependymoma (44-50\%) and less often in other tumors (medulloblastoma 7.1-13.3\%, astrocytoma $9 \%$ ). In our study, the frequency of calcification (ependymoma 66\%, medulloblastoma $11 \%$ and astrocytoma $12 \%$ ) was close to their reports. In this study, peritumoral edema was seen with decreasing frequency in medulloblastomas, ependymomas, cerebellar astrocytomas and brain steam gliomas. These findings were similar to the reports of Chang et al. ${ }^{12}$.

In this study it was found that measures of diagnostic performance of $\mathrm{CT}$ in different posterior fossa tumours are high with the overall accuracy of $\mathrm{CT}$ as diagnostic modality ranged between $93 \%$ and $100 \%$. From this present finding, it is conceivable that $\mathrm{CT}$ is one of the accurate diagnostic modality in evaluation of pediatric posterior fossa tumours.

\section{Conclusion:}

CT scan is useful modality in the evaluation of pediatric posterior fossa tumours. It can be regarded as a primary imaging modality in the diagnosis of different pediatric posterior fossa tumour and validated the related previous study findings regarding its efficacy. However, further study with larger study population involving several investigators at multiple centers may give more precise results.

\section{References:}

1. Osborn AG. Diagnostic neuroradiology. USA: Mosby; 1994: p.406-7.

2. Girolami UD, Anthony DC, Prosch MP. The central nervous system. In: Cotran RS, Kumar V, Collins T, eds. Robbins Pathologic Basis of Disease. $6^{\text {th }}$ ed. Philadelphia: WB Saunders; 1999: p. 1343 .

3. Manzione JV, Poe LB, Kieffer SA. Intracranial neoplasms. In: Hagga JR, Lanzieri CF, Sartoris DJ, Serhouni EA, eds. Computed Tomography and Magnetic Resonance Imaging of the whole body. $4^{\text {th }}$ ed. USA: Mosby; 2003: p.571-619.

4. Zimmerman RA, Bilaniuk LT, Pahlajani $\mathrm{H}$. Spectrum of medulloblastomas demonstrated by computed tomography. Radiology 1978; 126(1): 137-41.

5. Zimmerman RA, Bilaniuk LT, Bruno L, Rosenstock J. Computed Tomography of cerebellar astrocytoma. Am J Roentgenol 1978; 130(5): 92933.

6. Reider-Groswasser I, Catz A, Harel S. Posterior fossa lesions in childhood and infancy- a CT study. Child's Nerv Syst 1987; 3(4): 213-8.

7. Reider-Groswasser I, Catz A, Harel S. Computerized tomography findings in pediatric patients with posterior fossa lesions. Isr J Med Sci 1988; 24(4-5): 212-7.

8. Kingsley DP, Kendall BE. The CT scanner in posterior fossa tumours of childhood. Br J Radiol 1979; 52(622): 769-76.

9. Kendall BE, Kingsley DP. The value of computer axial tomography (CAT) in cranio-cerebral malformations. Br J Radiol 1978; 51(603): 171-90.

10. Zakrzewski K, Fiks T, Polis L, Liberski PP. Posterior fossa tumors in children and adolescents: a clinicopathological study of 216 cases. Folia Neuropathol 2003; 41(4): 251-2.

11. Bilaniuk LT, Zimmerman RA, Littman P, Gallo E, Rorke LB, Bruce DA, et al. Computed tomography of brain stem gliomas in children. Radiology 1980; 134(1): 89-95. 
12. Chang T, Teng MM, Lirng JF. Posterior cranial fossa tumours in childhood. Neuroradiology 1993; 35(4): 274-8.

13. Segall HD, Zee CS, Naidich TP, Ahmadi J, Becker TS. Computed tomography in neoplasms of the posterior fossa in children. Radiol Clin North Am 1982; 20(1): 237-53.
14. Naidich TP, Zimmerman RA. Primary brain tumors in children. Semin Roentgenol 1984; 19(2): 100-14.

15. Lee YY. Glass JP, van Eys J, Wallace S. Medulloblastoma in infants and children: computed tomographic follow-up after treatment. Radiology 1985; 154(3): 677-82.

16. Weisberg LA. Computed tomographic findings in medulloblastomas. Comput Radiol 1982; 6(2): 83-91. 